K. Miyake

Nagoya Math. J.

Vol. 80 (1980), 117-127

\title{
ON THE STRUCTURE OF THE IDELE GROUP OF AN ALGEBRAIC NUMBER FIELD
}

\author{
KATSUYA MIYAKE
}

The purpose of this paper is to present the results of E. Artin and Furtwängler, with which they proved the principal ideal theorem, as a structure theorem of the idele group of an algebraic number field. Such treatment may be helpful to clarify the Arithmetic nature these results possess.

$\S 1$.

Let $F$ be an algebraic number field (of finite degree over $\boldsymbol{Q}$ ), and let $K / F$ and $L / K$ be both finite abelian extensions. Suppose that $L$ is a Galois extension of $F$, and that $K$ is the maximal abelian extension of $F$ contained in $L$. Then $G=\operatorname{Gal}(L / F)$ is metabelian, and $G^{\prime}=\operatorname{Gal}(L / K)$ is the commutator subgroup of $G$.

Let us denote the Artin maps of $K / F$ and $L / K$ by $[\cdot, K / F]$ and $[\cdot, L / K]$ respectively. That is, for a prime ideal $\mathfrak{p}$ of $F$ which is unramified in $K / F$, $[\mathfrak{p}, K / F]$ is the Frobenius automorphism of $\mathfrak{p}$ in $\operatorname{Gal}(K / F)$.

Let $\mathfrak{a}$ be an ideal of $F$. Then the extension of $a$ to an ideal of $K$ is $\mathfrak{a} \cdot O_{K}$ where $O_{K}$ is the maximal order of $K$.

Theorem (Artin-Furtwängler). Let $L$ be a Galois extension of $F$, and suppose that $G=\mathrm{Gal}(L / F)$ is metabelian. Let $K$ be the maximal abelian extension of $F$ contained in $L$, and $O_{K}$ the maximal order of $K$. Then, if an ideal $\mathfrak{a}$ of $F$ is unramified in $K / F,\left[\mathfrak{a} \cdot O_{K}, L / K\right]$ is trivial.

E. Artin showed that the map of $G / G^{\prime}=\operatorname{Gal}(K / F)$ to $G^{\prime}=\operatorname{Gal}(L / K)$ which gives

$$
[\mathfrak{a}, K / F] \longmapsto\left[\mathfrak{a} \cdot O_{K}, L / K\right]
$$

is the transfer (Verlagerung) $V_{G \rightarrow G^{\prime}}$ of $G / G^{\prime}$ to $G^{\prime}$. Then Furtwängler proved that $V_{G \rightarrow G^{\prime}}$ is the trivial homomorphism of $G / G^{\prime}$ to $G^{\prime}$. (See [1] and [3].)

Received May 8, 1979. 
It may be worth to point out that this theorem is proved without using class field theory.

$\S 2$.

For an algebraic number field $F$, the ring of adeles of $F$ is denoted by $F_{A}$, and the idele group of $F$ by $F_{A}^{\times}$. Let $F_{a b}$ be the maximal abelian extension in the algebraic closure $\bar{F}$ of $F$, and put $\mathfrak{A}_{F}=\operatorname{Gal}\left(F_{a b} / F\right)$ and $\mathbb{G}_{F}=\operatorname{Gal}(\bar{F} / F)$. Let $F_{A}^{\times}=F_{f}^{\times} \cdot F_{\infty}^{\times}$be the decomposition of $F_{A}^{\times}$into the product of its non-Archimedean part $F_{f}^{\times}$and its Archimedean part $F_{\infty}^{\times}$. Let $F_{\infty+}^{\times}$be the connected component of the unity of $F_{\infty}^{\times}$, and $F^{\#}$ the topological closure of $F^{\times} \cdot F_{\infty+}^{\times}$in $F_{A}^{\times}$. Here and after, $F$ and $F^{\times}$are considered to be diagonally embedded in $F_{A}$ and $F_{A}^{\times}$respectively.

By class field theory, Artin map or canonical morphism

$$
[\cdot, F]: F_{A}^{\times} \longrightarrow \mathfrak{U}_{F}
$$

is an open, continuous and surjective homomorphism whose kernel is $F^{\#}$. Our basic reference on class field theory is Weil's book [8] though the notation slightly differs.

Let $K$ be a finite Galois extension of $F$. Then $\operatorname{Gal}(K / F)=\mathbb{S}_{F} / \mathscr{S}_{K}$ where $\mathbb{B S}_{K}=\mathrm{Gal}(\bar{F} / K)$. The ring of adeles of $K$ is naturally identified with the tensor product $K \otimes_{F} F_{A}=K_{A}$. Then the natural action of $\mathbb{S}_{F}$ on $K_{A}$ is the one defined through the $K$-factor of the product.

Let $\mathscr{S}_{K}^{\prime}$ be the commutator subgroup of $\mathscr{G}_{K}$. Then $\mathfrak{A}_{K}=\operatorname{Gal}\left(K_{a b} / K\right)=$ $\mathbb{S}_{K} / \mathbb{S}_{K}^{\prime}$. Since $\mathbb{S}_{K}$ is a normal subgroup of $\mathbb{S}_{F}$, this $\mathbb{S}_{F}$ acts on $\mathbb{S}_{K}$ through inner automorphisms of $\mathbb{S}_{F}$, and also on $\mathfrak{A}_{K}=\mathbb{S}_{K} / \mathbb{S}_{K}^{\prime}$. More precisely, let $\xi$ be an element of $\mathscr{S}_{K}$. Then for $\lambda \in \mathscr{G S}_{F}$, the action of $\lambda$ on $\xi \bmod \mathscr{S}_{K}^{\prime}$ is defined by

$$
\left(\xi \bmod \mathscr{S}_{K}^{\prime}\right)^{\lambda}=\lambda^{-1} \cdot \xi \cdot \lambda \bmod \mathscr{G}_{K}^{\prime} \cdot
$$

TheOREM 1. For $x \in K_{A}^{\times}$and $\lambda \in \mathbb{S}_{F}$,

$$
\left[x^{\lambda}, K\right]=[x, K]^{\lambda}
$$

where $[\cdot, K]: K_{A}^{\times} \rightarrow \mathfrak{U}_{K}=\operatorname{Gal}\left(K_{a b} / K\right)$ is Artin map for $K$.

This theorem is well known. But a proof will be given in $\S 6$ for the completeness.

$\S 3$.

Now our intended result is ready to be shown. Generalization will 
be done in the next section. Note that $K$ does not have to be an abelian extension of $F$ in this theorem.

Theorem 2. Let $F$ be an algebraic number field and $K$ a finite Galois extension of $F$. If an open subgroup $U$ of $K_{A}^{\times}$satisfies

(i) $U \supset K^{\#}$

(ii) $U^{o}=U$ for any $\sigma \in \operatorname{Gal}(K / F)$

(iii) $U \cdot N_{K / F}^{-1}\left(F^{\sharp}\right)=K_{A}^{\times}$

then $U \supset F_{A}^{\times}$.

Here $N_{K / F}: K_{A}^{\times} \rightarrow F_{A}^{\times}$is the norm map of $K$ over $F$.

Proof. First we reduce the theorem to the case that $K$ is an abelian extension of $F$. Let $M$ be the maximal abelian extension of $F$ contained in $K$. Then

$$
F^{\times} \cdot N_{M / F}\left(M_{A}^{\times}\right)=F^{\times} \cdot N_{K / F}\left(K_{A}^{\times}\right) .
$$

Put $V=M^{\times} \cdot N_{K / M}(U)$. Then $V$ is an open subgroup of $M_{A}^{\times}$, and contains $M^{*}$. It is obvious that $V^{\tau}=V$ for $\tau \in \operatorname{Gal}(M / F)$. Since

$$
F^{\times} \cdot N_{M / F}(V)=F^{\times} \cdot N_{K / F}(U)=F^{\times} \cdot N_{K / F}\left(K_{A}^{\times}\right)=F^{\times} \cdot N_{M / F}\left(M_{A}^{\times}\right)
$$

it is easy to see that

$$
V \cdot N_{M / F}^{-1}\left(F^{\#}\right)=V \cdot N_{M / F}^{-1}\left(F^{\times}\right)=M_{A}^{\times} .
$$

It follows, moreover, from (i) and (ii) that $U$ contains $V$ as a subgroup. Hence it is sufficient to show that $V$ contains $F_{A}^{\times}$. Therefore we may assume that $K$ itself is an abelian extension of $F$.

Now let $L$ be the class field of $K$ corresponding to $U$. Then

$$
U=K^{\times} \cdot N_{L / K}\left(L_{A}^{\times}\right) .
$$

By Theorem 1, condition (ii) implies that $L$ is a Galois extension of $F$. From (iii), it follows that $K$ is the maximal abelian extension of $F$ contained in $L$.

For a prime ideal $\Re$ of $K$, let $O_{K, \Re}$ be the $\beta$-adic completion of $O_{K}$, and $O_{K, \Re}^{\times}$the group of units of $O_{K, \Re}$. Then $O_{K, \Re}^{\times}$is canonically regarded as a subgroup of $K_{A}^{\times}$. Since $U$ is open, the number of such prime ideals $\mathfrak{P}$ that $O_{K, \Re}^{\times} \varangle U$ is finite. Let $S$ be the set of all such prime ideals of $K$. For each $\mathfrak{B} \in S$, fix an integer $e(\mathfrak{B})$ such that

$$
1+\mathfrak{P}^{e(\mathfrak{\beta})} \cdot O_{K, \mathfrak{B}} \subset U
$$


and

$U_{S}=\prod_{\Re \in S} O_{K, \Re}^{\times} \times \prod_{\Re \in S}\left(1+\mathfrak{B}^{e(\mathfrak{R})} \cdot O_{K, \mathfrak{\beta}}\right) \times K_{\infty+}^{\times}$

$K_{A(S)}^{\times}=$the subgroup of $K_{A}^{\times}$generated by $U_{S}$ and all $K_{\mathfrak{P}}^{\times}$for $\mathfrak{P} \notin S$

$K_{S}^{\times}=K^{\times} \cap K_{\boldsymbol{A}(S)}^{\times}$

$\mathfrak{M}=\prod_{\mathfrak{B} \in S} \mathfrak{P}^{e(\mathfrak{B})} \times$ product of all infinite places of $K$

$I_{L}(S)=$ the group of ideals of $L$ prime to $\mathfrak{M}$

$I_{K}(S)=$ the group of ideals of $K$ prime to $\mathfrak{M}$

$\mathfrak{S}_{K}(M)=$ the Strahl ideal class group modulo $\mathfrak{M}$.

Here $K_{\mathfrak{P}}$ is the $\mathfrak{P}$-adic completion of $K$, and $K_{\mathfrak{P}}^{\times}$is its multiplicative group. For prime $\boldsymbol{P}$ of $L$, let $L_{\boldsymbol{P}}$ be the $\boldsymbol{P}$-adic completion, and $L_{\boldsymbol{P}}^{\times}$the multiplicative group of $L_{P}$. Put

$L_{\boldsymbol{A}(S)}^{\times}=$the subgroup of $L_{A}^{\times}$generated by $\prod_{\boldsymbol{P} \cap K \in S} O_{L, P}^{\times}$and all $L_{\boldsymbol{P}}^{\times}$for $\boldsymbol{P} \cap K \notin S$.

For idele $x$ of $K$ (resp. of $L$, of $F$ ), denote the corresponding ideal of $K$ (resp. of $L$, of $F$ ) by $\mathscr{I}_{K}(x)$ (resp. $\mathscr{I}_{L}(x), \mathscr{I}_{F}(x)$ ). Then we have exact sequences

$$
\begin{aligned}
& 1 \longrightarrow U_{S} \longrightarrow K_{A(S)}^{\times} \stackrel{\mathscr{I}_{K}}{\longrightarrow} I_{K}(S) \longrightarrow 1 \\
& 1 \longrightarrow K_{S}^{\times} \cdot U_{S} \longrightarrow K_{A(S)}^{\longrightarrow} \longrightarrow \Im_{K}(S) \longrightarrow 1 \\
& L_{A(S)}^{\times} \cap N_{L / K}^{-1}\left(K_{A(S)}^{\times}\right) \stackrel{\mathscr{I}_{L}}{\longrightarrow} I_{L}(S) \longrightarrow 1 .
\end{aligned}
$$

Furthermore, for $x \in L_{A(S)}^{\times} \cap N_{L / K}^{-1}\left(K_{A(S)}^{\times}\right)$,

$$
\mathscr{I}_{K}\left(N_{L / K}(x)\right)=N_{L / K}\left(\mathscr{I}_{L}(x)\right)
$$

and, for $x \in F_{A}^{\times} \cap K_{A(S)}^{\times}$,

$$
\mathscr{I}_{K}(x)=\mathscr{I}_{F}(x) \cdot O_{K}
$$

Now apply Artin-Furtwängler theorem to this case. Then, (by Hilbert theory), one can easily conclude that, for $x \in F_{\boldsymbol{A}}^{\times} \cap K_{\boldsymbol{A}(S)}^{\times}$, there exist $a \in$ $K_{S}^{\times}$and $y \in L_{\boldsymbol{A}(S)}^{\times} \cap N_{L / K}^{-1}\left(K_{\boldsymbol{A}(S)}^{\times}\right)$such that

$$
\mathscr{I}_{K}(x)=\mathscr{I}_{K}(a) \cdot N_{L / K}\left(\mathscr{I}_{L}(y)\right) \text {. }
$$

Therefore

$$
x=a \cdot N_{L / K}(y) \cdot u
$$

with some $u \in U_{S}$. Since $U$ contains all of $K_{S}^{\times}, N_{L / K}\left(L_{A}^{\times}\right)$and $U_{S}$, it has 
been shown that

$$
F_{A}^{\times} \cap K_{A(S)}^{\times} \subset U
$$

Because $S$ is a finite set of prime ideals of $K$, one can easily see by Chinese remainder theorem that $\left(F_{A}^{\times} \cap K_{A(S)}^{\times}\right) \cdot F^{\times}=F_{A}^{\times}$. Since $U$ contains $F^{\times}$,

$$
F_{A}^{\times}=\left(F_{A}^{\times} \cap K_{A(S)}^{\times}\right) \cdot F^{\times} \subset U \cdot F^{\times}=U .
$$

The proof is done.

\section{§4. Generalization}

THEOREM 3. Let $F$ be an algebraic number field, and $K$ a finite Galois extension of $F$. For an open subgroup $U$ of $K_{A}^{\times}$satisfying

(i) $U \supset K^{\#}$

(ii) $U^{\sigma}=U$ for any $\sigma \in \mathrm{Gal}(K / F)$

put $m=\left[K_{A}^{\times}: U \cdot N_{K / F}^{-1}\left(F^{\#}\right)\right]$. Then

$$
\left(F_{\boldsymbol{A}}^{\times}\right)^{m}=\left\{a^{m} \mid a \in F_{\boldsymbol{A}}^{\times}\right\} \subset U .
$$

Proof. Let $L$ be the abelian extension of $K$ corresponding to $U \cdot N_{K / F}^{-1}\left(F^{\#}\right)$. Then $m=[L: K]$, and

$$
K^{\times} \cdot N_{L / K}\left(L_{A}^{\times}\right)=U \cdot N_{K / F}^{-1}\left(F^{\#}\right) .
$$

Put $V=N_{L / K}^{-1}(U)$. Then

$$
L_{\boldsymbol{A}}^{\times}=V \cdot N_{L / F}^{-1}\left(F^{\sharp}\right)
$$

since

$$
\begin{aligned}
F^{\times} \cdot N_{L / F}\left(L_{A}^{\times}\right) & =F^{\times} \cdot N_{K / F}\left(K^{\times} \cdot N_{L / K}\left(L_{A}^{\times}\right)\right) \\
& =F^{\times} \cdot N_{K / F}\left(U \cdot N_{K / F}^{-1}\left(F^{\sharp}\right)\right) \\
& =F^{\times} \cdot N_{K / F}(U) \\
& =F^{\times} \cdot N_{L / F}(V) .
\end{aligned}
$$

Obviously $L$ is a Galois extension of $F$. Theorem 2, therefore, is applicable to $L / F$ and $V$, and implies that $V \supset F_{A}^{\times}$. Hence for any $a \in F_{A}^{\times}$

$$
a^{m}=N_{L / K}(a) \in U \text {. }
$$

The proof is completed.

CoRollary. The notation and the assumptions being as in the theorem, let $n$ be the largest common divisor of $m$ and the degree $[K: F]$. Then 


$$
\left(U \cdot N_{K / F}^{-1}\left(F^{\#}\right)\right) \cap F_{A}^{\times}=(U \cdot X) \cap F_{A}^{\times}
$$

where $X=\left\{x \in N_{K / F}^{-1}\left(F^{*}\right) \mid x^{n} \in U\right\}$.

Therefore especially

$$
\left(U \cdot N_{K / F}^{-1}\left(F^{\#}\right)\right) \cap F_{A}^{\times}=U \cap F_{A}^{\times}
$$

if $n$ is prime to the index $\left[U \cdot N_{K / F}^{-1}\left(F^{*}\right): U\right]$.

Proof. Put $d=[K: F]$. For $a \in\left(U \cdot N_{K / F}^{-1}\left(F^{\sharp}\right)\right) \cap F_{A}^{\times}$, choose $u \in U$ and $v \in N_{K / F}^{-1}\left(F^{\#}\right)$ so that $a=u \cdot v$. Then $a^{d}=N_{K / F}(a)=N_{K / F}(u) \cdot N_{K / F}(v)$. Condition (ii) implies that $N_{K / F}(u) \in U$. Since $N_{K / F}(v) \in F^{\#}$, we conclude that $a^{d} \in U \cap F_{\boldsymbol{A}}^{\times}$. It follows from the theorem that $a^{m}$ belongs to $U \cap F_{\boldsymbol{A}}^{\times}$. Therefore $a^{n}$ belongs to $U \cap F_{A}^{\times}$where $n=(m, d)$. Since $a^{n}=u^{n} \cdot v^{n}$, we see that $v \in X$. The proof is done.

\section{§5. Remarks on $F^{\#}$}

Let $F$ be an algebraic number field of finite degree $d$ over $\boldsymbol{Q}$, and $d=r_{1}+2 \cdot r_{2}$ where $r_{1}$ is the number of real Archimedean primes of $F$. Put $r=r_{1}+r_{2}-1$. Let $E_{+}$be the multiplicative group of all the totally positive units of $F$. (We exclude the roots of 1 in $F$ from $E_{+}$when $r_{1}=$ 0 .) Then $E_{+}$is a free $Z$-module of rank $r$.

Let $E_{+f}$ be the projection of $E_{+}$to the non-Archimedean part $F_{f}^{\times}$of $F_{A}^{\times}$, and $\overline{E_{+f}}$ the topological closure of $E_{+f}$ in $F_{f}^{\times}$.

Proposition 1. The closure $F^{\#}$ of $F^{\times} \cdot F_{\infty+}^{\times}$in $F_{A}^{\times}$is equal to $\overline{E_{+f}} \cdot F^{\times}$. $F_{\infty++}^{\times}$. Moreover, for every positive integer $n$,

$$
\begin{gathered}
\overline{E_{+f}}=E_{+f} \cdot\left\{x^{n} \mid x \in \overline{E_{+f}}\right\} \\
F^{\#}=F^{\times} \cdot\left\{x^{n} \mid x \in F^{\sharp}\right\} .
\end{gathered}
$$

(See Shimura [7], 2.2.)

Proposition 2. (1) $F^{\times} \cap\left\{x^{n} \mid x \in F^{\#}\right\}=\left\{a^{n} \mid a \in F^{\times}\right\}$.

(2) For $x \in F^{\#}, x^{n}=1 \Rightarrow x \in F^{\times} \cdot F_{\infty+}^{\times}$.

(See [6], 3.1.)

Proposition 3. As topological groups, $\overline{E_{+f}}$ is isomorphic to the direct product of $r$ copies of $\tilde{Z}=\prod_{p \text {,prime }} Z_{p}$ where $Z_{p}$ is the ring of p-adic integers.

Proof. By Chevalley [2], the topology induced on free $Z$-module $E_{+f}$ of rank $r$ is the one defined by taking all the subgroups of finite index 
as the basis of the neighbourhood of 0 . Therefore $\overline{E_{+f}}$ is isomorphic to the completion $\tilde{\boldsymbol{Z}}^{r}$.

Proposition 4. Let $K$ be a finite extension of $F$ (not necessarily Galois). Then

$$
N_{K / F}^{-1}\left(F^{\#}\right) / K^{\#} \cdot N_{K / F}^{-1}(1) \cong N_{K / F}\left(K_{A}^{\times}\right) \cap F^{\times} / N_{K / F}\left(K^{\times}\right) .
$$

Proof. Put $N=N_{K / F}$, and $d=[K: F]$. First we see $N^{-1}\left(F^{\sharp}\right)=N^{-1}\left(F^{\times}\right)$ . $F^{\#}$. For $x \in N^{-1}\left(F^{\#}\right)$, choose $a \in F^{\times}$and $b \in F^{\#}$ by Prop. 1 so that $N(x)=$ $a \cdot b^{d}$. Put $y=x \cdot b^{-1}$. Then $N(y)=a \in F^{\times}$, and $x=y \cdot b$.

Next we show $N^{-1}\left(F^{\times}\right) \cap K^{\sharp}=K^{\times} \cdot\left(N^{-1}(1) \cap K^{\sharp}\right)$. Obviously the right is contained by the left. For $z \in K^{\#}$, suppose that $N(z) \in F^{\times}$. By Prop. 1 for $K$, choose $u \in K^{\times}$and $v \in K^{\#}$ so that $z=u \cdot v^{d}$. Then $N(v)^{d}=N(z) \cdot N(u)^{-1}$ $\in F^{\times}$. Therefore by Prop. 2, (1), we can find $a \in F^{\times}$such that $N(v)^{d}=a^{d}$. Then $z=(u \cdot a) \cdot\left(a^{-1} \cdot v^{d}\right)$ with $u \cdot a \in K^{\times}$and $N\left(a^{-1} v^{d}\right)=1$. Now

$$
\begin{aligned}
N^{-1}\left(F^{\sharp}\right) / K^{\sharp} \cdot N^{-1}(1) & =N^{-1}\left(F^{\times}\right) \cdot F^{\sharp} / K^{\sharp} \cdot N^{-1}(1) \\
& \cong N^{-1}\left(F^{\times}\right) / N^{-1}\left(F^{\times}\right) \cap\left(K^{\sharp} \cdot N^{-1}(1)\right) \\
& =N^{-1}\left(F^{\times}\right) /\left(N^{-1}\left(F^{\times}\right) \cap K^{\sharp}\right) \cdot N^{-1}(1) \\
& =N^{-1}\left(F^{\times}\right) / K^{\times} \cdot N^{-1}(1) \\
& \cong N\left(K_{A}^{\times}\right) \cap F^{\times} / N\left(K^{\times}\right) .
\end{aligned}
$$

The proof is done.

\section{§6. Proof of Theorem 1}

Let $K$ be a finite Galois extension of an algebraic number field $F$. Let the notation and the situation be as in $\S 2$. We have to prove that canonical homomorphism $[\cdot, K]: K_{A}^{\times} \rightarrow \mathfrak{A}_{K}=\mathrm{Gal}\left(K_{a b} / K\right)$ of class field theory is compatible with the action of $\mathbb{S}_{F}=\operatorname{Gal}(\bar{F} / F)$ (modulo $\left.\mathbb{S}_{K}\right)$.

Let $\mathfrak{p}$ be a prime divisor of $F, F_{\mathfrak{p}}$ the completion of $F$ at $\mathfrak{p}$, and $\bar{F}_{\mathfrak{p}}$ the algebraic closure of $F_{p}$. Fix an isomorphism $\iota$ of $\bar{F}$ into a subfield $\iota(\bar{F})$ of $\bar{F}_{p}$, which is identical on $F$. Put $\tilde{K}=\iota(K) \cdot F_{p}$. This is a Galois extension of $F_{p}$. Put $\overparen{S_{p}}=\operatorname{Gal}\left(\bar{F}_{p} / F_{p}\right)$ and $\widetilde{S}=\operatorname{Gal}\left(\bar{F}_{p} / \tilde{K}\right)$. The latter is a normal subgroup of the former. Note that $\bar{F}_{\mathfrak{p}}=\iota(\bar{F}) \cdot F_{\mathfrak{p}}, F_{\mathfrak{p}, a b}=\iota\left(F_{a b}\right) \cdot F_{\mathfrak{p}}$, and $\tilde{K}_{a b}$ $=\iota\left(K_{a b}\right) \cdot \tilde{K}$ where $F_{\mathfrak{p}, a b}$ and $\tilde{K}_{a b}$ are the maximal abelian extension of $F_{\mathfrak{p}}$ and $\tilde{K}$ in $\bar{F}_{\mathfrak{p}}$ respectively. Hence the restriction of the action of $\mathbb{S}_{p}$ on

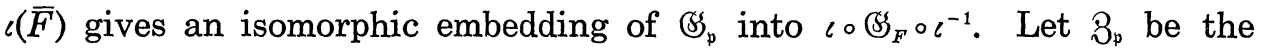
subgroup of $\mathscr{S}_{F}$ corresponding to $\mathbb{S}_{\mathfrak{p}}$. That is, $\iota \circ 3_{p} \circ \iota^{-1}=\mathbb{S}_{\mathfrak{p}}$. We also have 


$$
\begin{aligned}
& \mathscr{S}_{\mathfrak{p}}^{\prime}=\mathbb{S}_{\mathfrak{p}} \cap\left(\iota \circ \mathbb{S}_{F}^{\prime} \circ \iota^{-1}\right) \\
& \widetilde{\mathbb{S}}^{\prime}=\widetilde{\mathbb{S}} \cap\left(\iota \circ \mathbb{S}_{K}^{\prime} \circ \iota^{-1}\right)
\end{aligned}
$$

where $\mathscr{S}_{\mathfrak{p}}^{\prime}$ and $\widetilde{S^{\prime}}$ are the commutator subgroups of $\mathscr{S}_{\mathfrak{p}}$ and $\widetilde{\mathbb{S}}$ respectively.

Fix a set of representatives $S=\left\{\sigma_{1}, \cdots, \sigma_{g}\right\}$ of the left cosets of $\bigotimes_{p}$. (ङ) in $\mathscr{G}_{F}$. (Remember that $\mathscr{S}_{F}$ acts on both of $K_{A}$ and $\mathfrak{A}_{K}$ from the right.) For $\sigma \in \mathbb{S}_{F}$, the representative in $S$ of $B_{p} \cdot \mathscr{G}_{K} \cdot \sigma$ is denoted by $[\sigma]$. Put

$$
\iota(\sigma)=\iota \circ[\sigma]^{-1} \quad\left(\sigma \in \mathbb{S}_{F}\right) .
$$

Then $\iota(\sigma)$ depends only on the coset $\bigotimes_{p} \cdot \mho_{K} \cdot \sigma$. The family of pairs $\{(\iota(\sigma), K) \mid$ $\sigma \in S\}$ is a set of all non-equivalent proper embeddings of $K$ above $F_{p}$. That is, for any proper embedding $(\lambda, L)$ of $K$ above $F_{p}$, there are $\sigma \in S$ and isomorphism $\rho$ of $L$ over $F_{\mathfrak{p}}$ into $\tilde{K}$ such that $\iota(\sigma)=\rho \circ \lambda$. (See Weil [8], p. 51, Cor. 2.) Fix a set of representatives $R=\left\{\rho_{1}, \cdots, \rho_{f}\right\}$ of $\mathbb{\mathbb { S } _ { \mathfrak { p } }} / \widetilde{\mathbb{S}}=$ $\operatorname{Gal}\left(\tilde{K} / F_{p}\right)$ where $\rho_{i} \in \mathbb{B}_{p}$. Then for any two elements $\sigma, \tau$ of $\mathbb{S}_{F}$, there is a unique element $\rho(\sigma, \tau)$ of $R$ such that, restricted to $K$,

$$
\left.\iota(\sigma) \circ \tau\right|_{K}=\left.\rho(\sigma, \tau) \circ \iota\left(\sigma \tau^{-1}\right)\right|_{K} .
$$

For $\sigma$ and $\tau \in \mathbb{S}_{F}$, define $\zeta(\sigma, \tau) \in \mathbb{B}_{p} \cdot \mathscr{S}_{K}$ by

$$
[\sigma] \cdot \tau^{-1}=\zeta(\sigma, \tau) \cdot\left[\sigma \tau^{-1}\right] \text {. }
$$

Then

$$
\rho(\sigma, \tau) \equiv \iota \circ \zeta(\sigma, \tau)^{-1} \circ \iota^{-1} \quad \text { modulo } \widetilde{\mathscr{S}} \text {. }
$$

For each $\sigma \in S$, put

$$
\widetilde{\mathbb{S}_{\sigma}}=\sigma \circ \iota^{-1} \circ \widetilde{\mathbb{S}} \circ \iota \circ \sigma^{-1}=\iota^{-1} \circ\left[\left(\iota \circ \sigma^{-1} \circ \iota^{-1}\right) \cdot \widetilde{\mathbb{S}} \cdot\left(\iota \circ \sigma \circ \iota^{-1}\right)\right] \circ \iota .
$$

Then $\widetilde{\mathbb{S}_{o}}$ is a subgroup of $\mathscr{S}_{K}$ and is a conjugate of $\mathbb{Z}_{\mathfrak{p}} \cap \mathbb{S}_{K}$ in $\mathbb{S}_{F}$. It is easy to see that the commutator subgroup $\widetilde{\mathfrak{S}}_{\sigma}^{\prime}$ of $\widetilde{\mathbb{S}}_{\sigma}$ coincides with $\widetilde{\mathbb{S}}_{\sigma} \cap$ $\mathfrak{S}_{K}^{\prime}$. Put

$$
\mathfrak{U}_{\widetilde{K}, \sigma}=\widetilde{\mathbb{S}}_{\sigma} / \widetilde{\mathbb{S}}_{\sigma}^{\prime} .
$$

This is considered as a subgroup of $\mathfrak{U}_{K}=\mathbb{B}_{K} / \mathbb{S}_{K}^{\prime}$. The action of $\mathbb{B}_{F}$ on $\mathfrak{U}_{K}$ maps the family $\left\{\mathfrak{U}_{\tilde{K}, \sigma} \mid \sigma \in S\right\}$ onto itself. Each $\mathfrak{U}_{\tilde{K}, \sigma}$ is isomorphic to $\mathfrak{U}_{\tilde{K}}=\widetilde{\mathbb{S}} / \widetilde{\mathbb{S}}^{\prime}$.

Let us now consider the p-part of $K_{A}$. It is naturally identified with $K \bigotimes_{F} F_{p}$. Take copies of $\tilde{K}$ indexed by $S$. That is, put $\tilde{K}_{\sigma}=\tilde{K}$ for each $\sigma \in S$. Then the map $\iota(\sigma): K \rightarrow \tilde{K}_{\sigma}$ for $\sigma \in S$ gives an $F_{p}$-linear isomorphism 
$\eta_{\mathfrak{p}}$ of $K \otimes_{F} F_{\mathfrak{p}}$ onto the direct product $\prod_{\sigma \in S} \tilde{K}_{\sigma}$.

For $\sigma, \tau \in \mathbb{S}_{F}$, and for $a \in K$,

$$
\begin{aligned}
\iota(\sigma)\left(a^{\tau}\right) & =(\iota(\sigma) \circ \tau)(a)=\left(\rho(\sigma, \tau) \circ \iota\left(\sigma \tau^{-1}\right)\right)(a) \\
& =\left(\iota\left(\sigma \tau^{-1}\right)(a)\right)^{\rho(\sigma, \tau)} .
\end{aligned}
$$

Therefore it is easy to see the following:

$$
\text { For } x \in K \otimes_{F} F_{p}, \quad \text { let } \eta_{p}(x)=\left(x_{\sigma}\right)_{\sigma \in S} \in \prod_{\sigma} \tilde{K}_{\sigma} .
$$

Then for $\tau \in \mathscr{S}_{F}$,

$$
\begin{aligned}
\eta_{p}\left(x^{\tau}\right) & =\left(y_{\sigma}\right)_{\sigma \in S} \in \prod_{\sigma} \tilde{K}_{\sigma} \\
y_{\sigma} & =\left(x_{[\sigma \tau-1]}\right)^{\rho(\sigma, \tau)} .
\end{aligned}
$$

Let $\chi$ be a (linear) character of $\otimes_{K}$. It is automatically considered as a character of $\mathfrak{U}_{K}=\mathbb{S}_{K} / \mathbb{S}_{K}^{\prime}=\operatorname{Gal}\left(K_{a b} / K\right)$. For $\lambda \in \mathbb{S}_{F}$, define a character $\chi^{\lambda}$ of $\mathbb{S S}_{K}$ by

$$
\chi^{\lambda}(\tau)=\chi\left(\lambda \tau \lambda^{-1}\right) \quad\left(\tau \in \mathbb{S}_{K}\right) .
$$

Since $\mathbb{S}_{K}$ is normal in $\mathscr{S}_{F}$, this is well defined. Note that $\chi^{\lambda}$ depends only on $\lambda$ modulo $\mho_{K}$.

For $\chi$, we can associate characters $\chi_{\sigma}(\sigma \in S)$ of $\mathfrak{U}_{\tilde{K}}=\widetilde{\mathbb{S}} / \widetilde{\mathscr{S}}^{\prime}=\operatorname{Gal}\left(\tilde{K}_{a b} / \tilde{K}\right)$ through the isomorphisms of $\mathfrak{A}_{\tilde{K}}$ onto $\mathfrak{A}_{\tilde{K}, \sigma}$ established above. Namely for $\mu \in \widetilde{\mathbb{S}}$,

$$
\begin{aligned}
\chi_{\sigma}(\mu) & =\chi\left(\sigma \circ \iota^{-1} \circ \mu \circ \iota \circ \sigma^{-1}\right) \\
& =\chi\left(\sigma^{-1} \cdot\left(\iota^{-1} \circ \mu \circ \iota\right) \cdot \sigma\right) \\
& =\chi^{\sigma-1}\left(\iota^{-1} \circ \mu \circ \iota\right) .
\end{aligned}
$$

For a character $\chi$ of $\mathscr{\leftrightarrow}_{K}$, and for $x \in K \otimes_{F} F_{\mathfrak{p}}$ with $\eta_{\mathfrak{p}}(x)=\left(x_{\sigma}\right)_{\sigma \in S} \in \prod_{\sigma} \tilde{K}_{\sigma}$, the canonical pairing $(\chi, x)_{K, p}$ is defined by

$$
(\chi, x)_{K, p}=\prod_{\sigma \in S}\left(\chi_{\sigma}, x_{\sigma}\right)_{\tilde{K}},
$$

where each $\left(\chi_{\sigma}, x_{\sigma}\right)_{\tilde{K}}$ is the canonical pairing of local class field theory for $\tilde{K}_{\sigma}=\tilde{K}$.

Let $\lambda$ be an element of $\mathscr{B S}_{F}$. For $x \in K \otimes_{F} F_{\mathfrak{p}}$ with $\eta_{\mathfrak{p}}(x)=\left(x_{\sigma}\right)_{\sigma \in S}$, we had $\eta_{p}\left(x^{\lambda}\right)=\left(y_{\sigma}\right)$ with $y_{\sigma}=\left(x_{[\sigma \lambda-1]}\right)^{\rho(\sigma, \lambda)}$. On the other hand, $\left(\chi^{\lambda}\right)_{\sigma}(\mu)=$ $\chi^{\lambda \sigma^{-1}}\left(\iota^{-1} \circ \mu \circ \iota\right)$ for $\mu \in \widetilde{\mathbb{S}}$. Since $\sigma \lambda^{-1}=\zeta(\sigma, \lambda)\left[\sigma \lambda^{-1}\right]$,

$$
\left(\chi^{\lambda}\right)_{\sigma}(\mu)=\chi^{[\sigma \lambda-1]-1 \zeta(\sigma, \lambda)-1}\left(\iota^{-1} \circ \mu \circ \iota\right)
$$




$$
\begin{aligned}
& =\chi^{[\sigma \lambda-1]-1}\left(\zeta(\sigma, \lambda)^{-1} \cdot\left(\iota^{-1} \circ \mu \circ \iota\right) \cdot \zeta(\sigma, \lambda)\right) \\
& =\chi^{[\sigma \lambda-1]-1}\left(\zeta(\sigma, \lambda) \circ \iota^{-1} \circ \mu \circ \iota \circ \zeta(\sigma, \lambda)^{-1}\right) \\
& =\chi^{[\sigma \lambda-1]-1}\left(\iota^{-1} \circ \rho(\sigma, \lambda)^{-1} \circ \mu \circ \rho(\sigma, \lambda) \circ \iota\right) \\
& =\chi_{[\sigma \lambda-1]}\left(\rho(\sigma, \lambda)^{-1} \circ \mu \circ \rho(\sigma, \lambda)\right) \\
& =\chi_{[\sigma \lambda-1]}\left(\rho(\sigma, \lambda) \cdot \mu \cdot \rho(\sigma, \lambda)^{-1}\right) \\
& =\left(\chi_{[\sigma \lambda-1]}\right)^{\rho(\sigma, \lambda)}(\mu) .
\end{aligned}
$$

Therefore

$$
\begin{aligned}
\left(\chi^{\lambda}, x^{\lambda}\right)_{K, \downarrow} & =\prod_{\sigma \in S}\left(\left(\chi^{\lambda}\right)_{\sigma}, y_{\sigma}\right)_{\tilde{K}} \\
& =\prod_{\sigma \in S}\left(\left(\chi_{[\sigma \lambda-1]}\right)^{\rho(\sigma, \lambda)},\left(x_{[\sigma \lambda-1]}\right)^{\rho(\sigma, \lambda)}\right)_{\tilde{K}} .
\end{aligned}
$$

Since $\rho(\sigma, \lambda) \in \mathbb{S}_{p}=\operatorname{Gal}\left(\bar{F}_{p} / F_{p}\right)$, and since $\tilde{K}$ is a Galois extension of $F_{p}$, we have $\tilde{K}^{\rho(\sigma, \lambda)}=\tilde{K}$.

Therefore

$$
\left(\left(\chi_{[\sigma \lambda-1]}\right)^{\rho(\sigma, \lambda)},\left(x_{[\sigma \lambda-1]}\right)^{\rho(\sigma, \lambda)}\right)_{\tilde{K}}=\left(\chi_{[\sigma \lambda-1]}, x_{[\sigma \lambda-1]}\right)_{\tilde{K}} \text {. }
$$

(See Weil [8], p 223, Cor. 5.) This shows that

$$
\left(\chi^{2}, x^{2}\right)_{K, \mathfrak{p}}=(\chi, x)_{K, \mathfrak{p}} .
$$

Since this is true for any prime divisor of $F$,

$$
\left(\chi^{\lambda}, x^{\lambda}\right)_{K}=(\chi, x)_{K}
$$

for $x \in K_{A}^{\times}, \lambda \in \mathbb{S}_{F}$ and a character $\chi$ of $\mathbb{S}_{K}$. Here $(\chi, x)_{K}$ is the canonical pairing of $K$.

The canonical morphism

$$
[\cdot, K]: K_{A}^{\times} \longrightarrow \mathfrak{U}_{K}=\mathscr{\mho}_{K} / \mathscr{S}_{K}^{\prime}=\operatorname{Gal}\left(K_{a b} / K\right)
$$

is defined so that

$$
(\chi, x)_{K}=\chi([x, K])
$$

for any $x \in K_{A}^{\times}$and any $\chi$. For each $[x, K] \in \mathfrak{A}_{K}$, choose $[x, K]^{*} \in \mathbb{S}_{K}$ so that $[x, K]^{*}$ modulo $\mathbb{F}_{K}^{\prime}$ is $[x, K]$. Then for $\lambda \in \mathbb{S}_{F}$,

$$
\left(\chi^{\lambda}, x^{\lambda}\right)_{K}=\chi^{\lambda}\left(\left[x^{\lambda}, K\right]^{*}\right)=\chi\left(\lambda \cdot\left[x^{\lambda}, K\right]^{*} \cdot \lambda^{-1}\right) .
$$

Therefore

$$
\chi([x, K])=\chi\left(\lambda \cdot\left[x^{\lambda}, K\right]^{*} \cdot \lambda^{-1}\right)
$$

for any $\chi$. This implies that 


$$
[x, K]=\lambda \cdot\left[x^{\lambda}, K\right]^{*} \cdot \lambda^{-1} \text { modulo } \mathfrak{G S}_{K}^{\prime} .
$$

Equivalent to say,

$$
\lambda^{-1} \cdot[x, K]^{*} \cdot \lambda \equiv\left[x^{2}, K\right]^{*} \quad \text { modulo } \mathbb{G S}_{K}^{\prime} \text {. }
$$

This is what Theorem 1 claims. The proof is done.

\section{REFERENCE}

[1] E. Artin, Idealklassen in Oberkörpern und allgemeine Reziprozitätsgesetze, Abh. Math. Sem. Hamburg 7 (1930).

[2] C. Chevalley, Deux théorèmes d'arithmétique, J. Math. Soc. Japan 3 (1951).

[ 3 ] Ph. Furtwängler, Beweis des Hauptidealsatzes für Klassenkörper algebraischer Zahlkörper, Abh. Math. Sem. Hamburg 7 (1930).

[4] J. Herbrand, Sur les théorèmes du genre principal et des ideaux principaux, Abh. Math. Sem. Hamburg 9 (1933).

[5] S. Iyanaga, Ưber den allgemeinen Hauptidealsatz, Jap. J. Math. 7 (1930).

[6] K. Miyake, Models of certain automorphic function fields, Acta Math. 126 (1971).

[7] G. Shimura, On canonical models of arithmetic quotient of bounded symmetric domains II, Ann. of Math. 92 (1970).

[ 8 ] A. Weil, Basic Number Theory, Springer-Verlag, Berlin (1967).

Nagoya University 